\title{
The Effects of an Increase in Intensity during Tapering on 1,500m Running Performance
}

Kate L. Spilsbury ${ }^{\mathrm{a}, \mathrm{b}}$, Myra A. Nimmo $^{\mathrm{b}}$, Barry W. Fudge ${ }^{\mathrm{c}}$, Jamie S.M. Pringle ${ }^{\mathrm{a}}$, Mark W. Orme ${ }^{\mathrm{b}}$ and Steve H. Faulkner ${ }^{\mathrm{b}}$,

\section{Affiliations}

aEnglish Institute of Sport, Loughborough, UK

${ }^{\mathrm{b}}$ School of Sport, Exercise and Health Sciences, Loughborough University, UK

${ }^{\mathrm{c} B r i t i s h}$ Athletics, Loughborough, UK

\section{Correspondence:}

Dr Steve H. Faulkner, Department of Engineering, Nottingham Trent University, Clifton Lane, Clifton, Nottingham, NG11 8NS, UK, Phone: +44 (0)115 848 6196, Email: steve.faulkner@ntu.ac.uk

\section{Current Affiliations:}

Dr Kate L. Spilsbury; School of Public Health, The University of Queensland, Brisbane, 4006, Australia Prof Myra A. Nimmo; College of Life and Environmental Sciences, University of Birmingham, Edgbaston, Birmingham, B15 2TT, UK

Dr Jamie S.M. Pringle; Boardman Performance Centre, The Valley, Evesham, WR11 4DS, UK

Dr Mark W. Orme; NIHR Leicester Biomedical Research Centre, Leicester General Hospital, Gwendolen Road, Leicester, LE5 4PW, UK

Dr Steve H. Faulkner; Department of Engineering, Nottingham Trent University, Clifton Lane, Clifton, Nottingham, NG11 8NS, UK 


\section{Abstract}

We examined the effect of completing the final interval training session during a taper at either:

i) race pace; or ii) faster than race pace, on 1,500 m running performance and neuromuscular performance. Ten trained runners (age $21.7 \pm 3.0$ years, height $182.9 \pm 7.0 \mathrm{~cm}$, body mass 73.4 $\pm 6.8 \mathrm{~kg}$, personal best 1,500 $\mathrm{m}$ time 4:17.5 \pm 0:26.9 min) completed two conditions, consisting of 7-d of regular training and a 7-d taper, separated by three weeks of training. In one condition, the taper was prescribed using prediction models based on the practices of elite British middledistance runners, with the intensity of the final interval session being equal to $1,500 \mathrm{~m}$ race pace (RP). The taper was repeated in the HI condition, except the final interval session was completed at $115 \%$ of $1,500 \mathrm{~m}$ race pace. A $1,500 \mathrm{~m}$ treadmill time trial, measures of maximum voluntary isometric strength (MVC) and rate of force development (RFD) were completed before and after regular training and tapering. Performance was most likely improved after RP (mean $\pm 90 \%$ confidence limits, $10.1 \pm 1.6 \mathrm{~s}$ ), and possibly beneficial after HI $(4.2 \pm 12.0 \mathrm{~s})$. Both MVC force $(p=0.002)$ and RFD $(p=0.02)$ were improved after tapering, without differences between conditions. A race-pace taper based on the practices of elite middledistance runners is recommended to improve performance in young, sub-elite runners. The effect of this strategy with an increase in interval intensity is highly variable and should be implemented with caution.

Key Words: taper, interval training, middle-distance, training load, athlete, running 


\section{Introduction}

During heavy phases of training, an accumulation of fatigue may mask physiological adaptations in elite endurance athletes and supress the ability to perform (Halson and Jeukendrup 2004). It is therefore common to undertake a period of modified training before competition, known as tapering. Tapering has been defined as "a progressive nonlinear reduction of the training load during a variable period of time, in an attempt to reduce the physiological and psychological stress of daily training and optimise sports performance" (Mujika and Padilla, 2000). Tapering can be achieved by manipulating the training load variables of volume, frequency and intensity over a given duration (Houmard 1991). Previous research has attempted to optimise tapering strategies, with reported performance improvements of 0.5-6.0\% (Mujika and Padilla 2003).

To deliver a successful taper, an approximate two-week reduction in training volume of 41-60\%, with maintenance of training frequency and intensity is recommended (Bosquet et al. 2007). Whilst a reduction in volume might be necessary to overcome accumulated fatigue, evidence supports the inclusion of high intensity training during the taper to improve endurance running performance (Shepley et al. 1992, Houmard et al. 1994, Bosquet et al. 2007, Mujika 2010). When volume is reduced substantially ( $85-90 \%)$ and all training during the taper is completed as high intensity intervals (100-500 m), amplified physiological responses including buffering capacity (Houmard et al. 1994), oxidative enzyme activity, red blood cell volume and muscle glycogen content (Shepley et al. 1992), are evident. In practice however, the tapering strategies of elite endurance athletes incorporate both high intensity interval training and lower intensity continuous running, with volume reduction being to a lesser extent (Stellingwerff 2012, Spilsbury et al. 2015). In elite skiers and biathletes, low intensity and high intensity training remained at a similar frequency from the pre-peaking phase to the final 14 days before major competition, however high intensity training progressed toward a more polarised model 
during this period (Tønnessen et al. 2014). Training data from the world's most successful female cross-country skier also confirmed the inclusion of low intensity training during the taper (Solli et al. 2017). This was accompanied by a progressive increase in the proportion of high versus moderate intensity training in the final three weeks before major competition and the inclusion of three high intensity sessions in the final seven days (Solli et al. 2017). In support of implementing a taper consisting of both high intensity short intervals $(100-500 \mathrm{~m}$ repetitions at $105 \% \dot{\mathrm{VO}}_{2} \mathrm{max}$ ) and low intensity continuous running, $10 \mathrm{~km}$ performance and $\dot{\mathrm{V}}{ }_{2}$ max were improved in trained long distance runners after four weeks, albeit at the expense of running economy (Munoz et al. 2015). However, there is a paucity of experimental studies recommending the best practices for high intensity training in the final days of the taper for optimising performance (Tønnessen et al. 2014).

The tapering strategies of elite British endurance runners have been explored in detail, and algorithms were developed, which predict an individual tapering protocol from the regular training load (Spilsbury et al. 2015). It was clear from these data that British long-distance and marathon runners train at intensities higher than average race pace within the final days of the taper period before competition, but this was not evident in middle-distance runners who train at race pace. The reason for this is uncertain, although it is possible that middle distance runners may not exceed race pace due to the volume of the training session, to familiarise themselves with race pace in preparation for the competition or through fear of increased injury risk (Spilsbury et al. 2015). However, an interval session completed faster than race pace late in the taper when the athlete is more fully recovered, might allow greater capacity to respond effectively to this type of training stimulus (Mujika et al. 2004) and further improve subsequent performance. In support, theoretical models have shown that a moderate increase in training load at the end of taper might further improve performance as the athlete can capitalise on additional adaptation, after initially overcoming accumulated fatigue from previous training 
(Thomas et al. 2009). Despite evidence of this practice in long-distance and marathon runners, it is not clear whether it would be of benefit to the performance of middle-distance runners.

The physiological mechanisms fundamental to the process of tapering have not yet been well defined in endurance runners. In swimmers, increased strength and power have been commonly observed as a result of tapering and such increases have been associated with improved performance (Cavanaugh and Musch 1989, Costill et al. 1985, Johns et al. 1992, Raglin et al. 1996). In endurance-trained runners however, the findings relating to neuromuscular performance are equivocal (Shepley et al. 1992, Houmard et al. 1994, Luden et al. 2010). This may be due to variances in participant training status, the type of tapering strategy undertaken and differences in the methodology implemented to measure force. Since improved neuromuscular performance is known to have a positive impact on the key determinants of performance in middle-distance events (Berryman et al. 2018, Blagrove et al. 2018), it is necessary to further investigate neuromuscular responses to both current and novel tapering strategies in middle-distance runners.

The primary aims of the current study were to: 1) investigate the effectiveness of an algorithm-derived tapering protocol on 1,500 m time trial performance; 2) establish whether an increase in the intensity of the final interval session (to $115 \%$ of race speed) during this tapering protocol can further enhance 1,500 m time trial performance, compared to the same session completed at race pace; 3) investigate the extent to which measures of neuromuscular performance may explain changes in performance in response to the two tapering strategies. 


\section{Materials \& methods}

\section{Participants}

Ten sub-elite trained male middle-distance runners; (mean \pm SD) age $21.7 \pm 3.0$ years, height $182.9 \pm 7.0 \mathrm{~cm}$, body mass $73.4 \pm 6.8 \mathrm{~kg}$, volunteered to take part in this study. Participants were competitive athletes $(800 \mathrm{~m} \& 1,500 \mathrm{~m})$, with a training history of at least two years and had trained consistently (including low intensity continuous training and high intensity interval training) without interruption for the previous two months. Personal best $1,500 \mathrm{~m}$ time was $($ mean \pm SD) 4:17.5 \pm 0:26.9 min (range; 3:51.3 - 5:16.7 min). Participants provided written informed consent to take part in the study, which was approved by the Institutional ethics committee and carried out in accordance with the Declaration of Helsinki.

\section{Experimental design}

The study employed a counterbalanced cross over study design (figure 1). Each of the two conditions involved a 7-d period of regular training (control) and a 7-d period of tapering and were separated by at least three weeks of regular training. Performance assessments were carried out on the day before the control period (day 1; baseline), the day after the control period (day 9; post-control) and on the day after the taper period (day 17; post-taper); totalling six performance trials. Participants were familiarised to the procedures before the study began. 


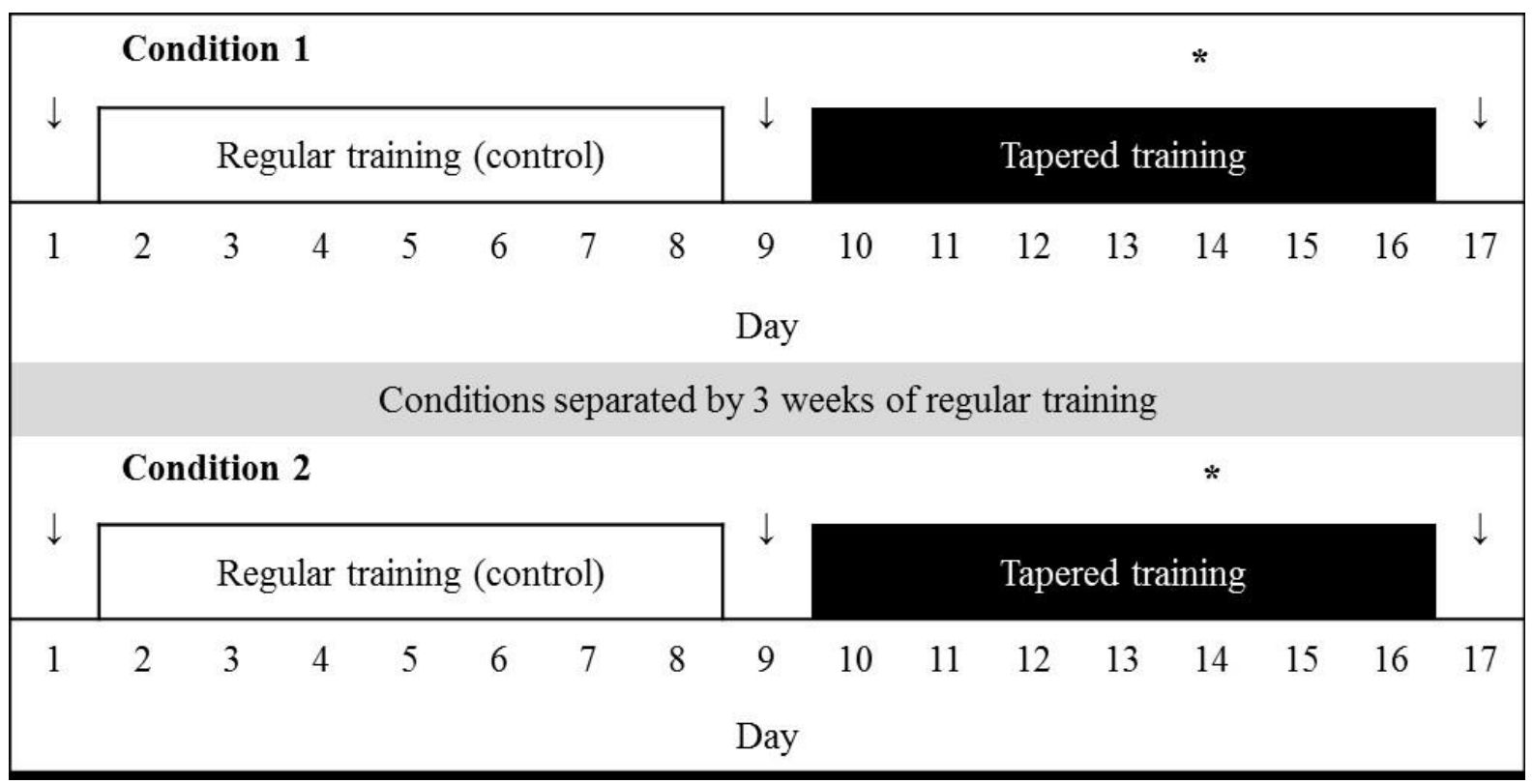

Figure 1. Study design illustrated by two experimental conditions, separated by at least three weeks of regular training. Arrows represent performance assessments; asterisk indicates laboratory interval session.

Training during the first control period was determined by the participant and recorded objectively from their own GPS data. Participants were instructed to replicate this training in the control period of the second condition. Training was categorised into continuous running (excluding warm up and warm down) or interval running and quantified for frequency, volume $(\mathrm{km})$ and duration $(\mathrm{min})$. For training intensity, mean speed was calculated from the volume and duration of each continuous run or interval repetition and expressed as a percentage of personal best $1,500 \mathrm{~m}$ race speed. During the taper period in both conditions, participants completed individualised training prescribed by predictive equations that consider control period training load (Spilsbury et al. 2015). An example of how this data was used to calculate tapered training load for an individual using the prediction models is shown in table 1, with the corresponding training program in table 2. After adjusting for the change in load, the general structure of the training program and specific interval sessions were replicated as closely as 
possible during the taper period. Participants were instructed to carry out the same warm and warm down for interval sessions as in the control period.

In the race-pace condition (RP), the final interval session of the taper was carried out at an intensity equivalent to average $1,500 \mathrm{~m}$ race speed. In the high-intensity condition (HI), training during the taper period was the same as RP, except the final interval session was performed at $115 \%$ of the speed in RP. This intensity was selected based on the practices of elite British (Spilsbury et al. 2015) and Kenyan (unpublished data) long distance and marathon runners. Participants were randomized to receive either the RP condition followed by the HI condition or the HI condition followed by the RP condition. Training was confirmed throughout all periods using GPS data. The investigation took place during the indoor and pre-outdoor competitive seasons (January-April). 
Table 1. An example of tapered training load calculated from control training load using prediction models developed from the tapering strategies in elite British endurance athletes*

\footnotetext{
Training Variables $\quad$ Example Data

\section{Continuous Volume}

Control (km) $\quad 65$

$\operatorname{Taper}^{a}(\%$ control $) \quad 65$

Taper $(\mathrm{km}) \quad 42$

Interval Volume ${ }^{\dagger}$

Control (km) $\quad 10$

$\operatorname{Taper}^{b}(\%$ control $) \quad 55$

Taper $(\mathrm{km}) \quad 6$

Continuous Frequency

$\begin{array}{rc}\text { Control }\left(\text { runs }^{\text {week }}{ }^{-1}\right) & 7 \\ \operatorname{Taper}^{c}(\% \text { control }) & 64 \\ \text { Taper }\left(\text { runs }^{\text {weeek }}{ }^{-1}\right) & 4\end{array}$

Interval Frequency

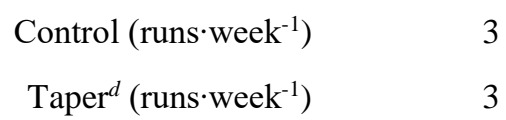

\section{Continuous Intensity}

$\begin{array}{rr}\text { Control }(\% \text { race speed }) & 60 \\ \operatorname{Taper}^{e}(\% \text { race speed }) & 57\end{array}$

Interval Intensity

$\begin{array}{rc}\text { Control }(\% \text { race speed }) & 96 \\ \operatorname{Taper}^{f}(\% \text { race speed }) & 100\end{array}$

*prediction models with control training load variables as predictors (Spilsbury et al. 2015).

${ }^{a}(97.153+(-0.106 *$ control continuous volume $)+(-2.547 *$ control continuous frequency $) * 0.9)$, adjusted for standard error of the estimate.

${ }^{b}$ average reported by British middle-distance runners (55\% of control interval volume).

${ }^{c}(130.800+(0.211 *$ control continuous volume $)+(1.059 *$ control interval volume $)+(-10.016 *$ control continuous frequency)).

${ }^{d}$ Control interval frequency maintained. Laboratory interval session was included in this frequency, not additional. ${ }^{e}(-13.443+(-0.07 *$ control continuous volume $)+(0.946 *$ control continuous frequency $)+(1.141 *$ control continuous intensity)).

${ }^{f}(34.356+(0.684 *$ control interval intensity $))$.

${ }^{\dagger}$ warm up and warm down volume for interval sessions not included.
} 
Table 2. An example of the individualised training completed during the control and taper period

\begin{tabular}{|c|c|c|}
\hline Day & Morning & Evening \\
\hline 1 & $1,500 \mathrm{~m}$ treadmill time trial & - \\
\hline \multicolumn{3}{|l|}{ Control } \\
\hline 2 & 20 km @60\% 1,500 m speed & - \\
\hline 3 & 4 x 1,000 m (120 s)@ 90\% 1,500 m speed & - \\
\hline 4 & 10 km @ 60\% 1,500 m speed & $5 \mathrm{~km} @ 60 \% 1,500 \mathrm{~m}$ speed \\
\hline 5 & $10 \mathrm{~km} @ 60 \% 1,500 \mathrm{~m}$ speed & 5 x $600 \mathrm{~m}$ (90 s)@ 95\% 1,500 m speed \\
\hline 6 & 10 km@60\% 1,500 m speed & $5 \mathrm{~km} @ 60 \% 1,500 \mathrm{~m}$ speed \\
\hline 7 & $\begin{array}{l}5 \times 400 \mathrm{~m}(60 \mathrm{~s}), 5 \times 200 \mathrm{~m}(45 \mathrm{~s}) @ 100 \% \text {, } \\
110 \% 1,500 \mathrm{~m} \text { speed }\end{array}$ & - \\
\hline 8 & Rest day & - \\
\hline 9 & $1,500 \mathrm{~m}$ treadmill time trial & - \\
\hline \multicolumn{3}{|l|}{ Taper } \\
\hline 10 & $15 \mathrm{~km} @ 57 \%$ 1,500 m speed & - \\
\hline 11 & 4 x 500 m (120 s)@ 100\% 1,500 m speed & - \\
\hline 12 & $9 \mathrm{~km} @ 57 \% 1,500 \mathrm{~m}$ speed & $\begin{array}{l}3 \text { x } 600 \text { m, } 1 \text { x 400m (90 s) @ 100\% 1,500 m } \\
\text { speed }\end{array}$ \\
\hline 13 & $9 \mathrm{~km} @ 57 \%$ 1,500 m speed & - \\
\hline 14 & $\begin{array}{l}6 \times 300 \text { m }(90 \mathrm{~s}) @ 100 \%(\mathrm{RP}) \text { or } 115 \%(\mathrm{HI}) \\
1,500 \mathrm{~m} \text { speed }\end{array}$ & - \\
\hline 15 & $9 \mathrm{~km} @ 57 \% 1,500 \mathrm{~m}$ speed & - \\
\hline 16 & Rest day & - \\
\hline 17 & $1,500 \mathrm{~m}$ treadmill time trial & - \\
\hline
\end{tabular}

Recovery interval duration shown in brackets; RP, race-pace condition; HI, high-intensity condition.

\section{Laboratory interval session within taper period}

An interval session was completed on a motorised treadmill (Woodway, Germany) on day 14, three days before the final performance assessment. Participants arrived fasted between 0700 and 0900, having completed only low intensity continuous running the day before. A standardised warm up was performed, consisting of 10 min running at a speed equivalent to $60 \%$ of personal best $1,500 \mathrm{~m}$ time, followed by two sets of $10 \mathrm{~s}$ at $90 \%$ and $20 \mathrm{~s}$ at $60 \%$ with 1-min rest between (Wiles et al. 1992). Afterwards, a series of $300 \mathrm{~m}$ interval repetitions with $90 \mathrm{~s}$ recovery was completed. The number of repetitions was individualised, depending upon 
interval volume calculated from the prediction equation. Intensity was equivalent to season's best $1,500 \mathrm{~m}$ race speed in the RP condition and $115 \%$ of season's best $1,500 \mathrm{~m}$ race speed in the HI condition. Heart rate was recorded (RS200, Polar Electro, Kempele, Finland) in the last $5 \mathrm{~s}$ of each repetition and rating of perceived exertion (RPE) immediately after each repetition. A cool down of 10 min running at $60 \%$ of $1500 \mathrm{~m}$ personal best race speed was performed.

\section{Performance assessments}

Participants arrived in a fasted state between $0700 \mathrm{~h}$ and $0900 \mathrm{~h}$ on days 1,9 and 17 of each condition. A rest day was prescribed the day before each performance assessment. Body mass and height were recorded, before assessments of muscle function. After a 30-min rest period, a 1,500 m treadmill time trial was completed.

\section{Force measurement}

Participants were strapped into an isometric strength rig, in a seated position to measure peak isometric voluntary knee extension (MVC) force. Knee angle was fixed at $60^{\circ}$ flexion and the ankle brace was $1 \mathrm{~cm}$ above the lateral malleolus on the right tibia. Participants placed their hands across their chest to further isolate the quadriceps contraction measurement and minimise upper body contribution. Eight sub-maximal contractions at intensities relative to perceived maximal force were performed to warm-up (3x 25\%, 2x 50\%, 2x 75\% and 1x 90\%), followed by 3-4 maximum contractions of $\sim 3-5 \mathrm{~s}$ duration and interspersed with $30 \mathrm{~s}$ rest periods (Tillin et al. 2010). Force was recorded using a calibrated S-beam strain gauge (01,500 N linear range; Force Logic, Swallowfield, UK) strapped to the distal region of the tibia. Force data were sampled and recorded at $5,000 \mathrm{~Hz}$ using an external A/D converter (Micro 1401, CED, Cambridge, UK) and a PC utilising Spike 2 software (CED, Cambridge, UK) and the peak force was used in data analysis. Subsequently, explosive isometric voluntary knee extensor contractions were performed to measure the rate of force development (RFD). 
Approximately 10 attempts of $\sim 1 \mathrm{~s}$ duration were required. Participants were instructed to develop force as quickly as possible from rest. Rest (20 s) was taken between attempts. The three explosive voluntary contractions displaying the highest peak RFD were selected for further analysis, and the results averaged across these three contractions. Force was measured at 50, 100 and $150 \mathrm{~ms}$ after force onset $\left(\mathrm{F}_{50}, \mathrm{~F}_{100}\right.$ and $\mathrm{F}_{150}$, respectively). Onset was defined as the last peak or trough before force exceeded the limits of the noise during the preceding 500 ms (Tillin et al. 2010). This systematic, manual identification of force onset has been shown to be both highly accurate (Allison 2003; Tillin et al. 2013) and reliable (Buckthorpe et al. 2012; Tillin et al. 2013).

\section{1,500 m performance assessment}

A $1,500 \mathrm{~m}$ treadmill time trial was completed after the standardised warm up on an 'onresponse' treadmill (MTC Climb 2000, Runner, Italy). A treadmill time trial was selected to eliminate the influence of different weather conditions associated with using an outdoor running track, and for the feasibility of completing the force measurements beforehand. Treadmill sensors allow the detection of user position on the belt and control belt velocity in accordance with user position relative to these sensors, allowing an autonomous speed adjustment. After a rolling start of $30 \mathrm{~s}$ at $60 \%$ of personal best time, participants completed a $1,500 \mathrm{~m}$ time trial, at a $1 \%$ gradient (Jones and Doust 1996). Speed and time indicators were concealed, but distance remained visible. Heart rate was recorded at 30-s intervals (RS200, Polar Electro, Kempele, Finland). Participants did not receive verbal encouragement or feedback. Prior to the main investigation, reliability of the 1,500 m performance assessment was tested. Time trials were performed fasted, on two separate occasions, seven days apart, after controlling for physical activity, diet and caffeine intake $24 \mathrm{~h}$ beforehand. The mean coefficient of variation $(\mathrm{CV})$ for time trial completion was $0.9 \%$, similar to the variation reported for $1,500 \mathrm{~m}$ track time trial performance $(0.8 \%)$ in well-trained runners (Hodges et al. 2006). 
The 'on response' treadmill time trial was therefore considered appropriate for use in the main investigation.

\section{Dietary intake and physical activity}

Dietary intake and physical activity were monitored throughout both conditions to assess consistency. Participants were instructed to eat and drink ad libitum during the control and taper of each condition and to weigh all food and fluid consumed (Salter Arc, Kent, UK). Total energy and carbohydrate intakes were calculated for each condition (CompEat Pro 5.8.0, Grantham, UK).

Physical activity was monitored using ActiGraph GT3X+ accelerometers (ActiGraph, Pensacola, FL) during the control and taper of RP and HI. Sampling frequency was $60 \mathrm{~Hz}$, analysed in $60 \mathrm{~s}$ epochs. A total of six monitors were used and each participant wore the same accelerometer throughout to minimise inter-device variability. Devices were fitted at the midline of the right anterior hip and worn daily from waking until sleep, except for water-based activities. Days with fewer than 600 min of wear time were not included. Non-wear time was defined as continuous runs of zeros lasting $\geq 60 \mathrm{~min}$, with no allowance for counts greater than zero. Cut-points to classify sedentary, light and moderate to vigorous physical activity (MVPA) were 0 - 99, 100 - 1951 and 1952 - 9498 counts per minute, respectively (Freedson et al. 1998). Average movement intensity was calculated using total average counts per minute. Time spent in sedentary, light and MVPA was calculated as a percentage of total wear time.

\section{Blood sampling}

After the time trials and controlled laboratory interval session, single capillary blood samples were obtained from the fingertip using an automated lancet at $0 \mathrm{~min}, 1 \mathrm{~min}$ and $2 \mathrm{~min}$ postcompletion for peak lactate estimation. An end-to-end capillary tube collected $20 \mu \mathrm{l}$ of blood 
which was transferred immediately into a polypropylene tube prefilled with $1 \mathrm{ml}$ of haemolysis solution, inverted and analysed using an automated device (Biosen C-Line, EKF Diagnostics, Barleben, Germany).

\section{Statistical analysis}

Data were analysed using SPSS 22.0 (Statistical Package for Social Sciences Inc., Chicago, IL). These data were tested for distribution and subsequently non-parametric tests were used where the data were not normally distributed, specifically energy intake in the final three days of the taper. Body mass and laboratory interval session data were compared using paired samples $t$-tests. Performance measures from day 1 and 9 were compared using a paired-samples $t$-test to ensure no-learning effect. No significant differences were evident (RP; $296 \pm 20 \mathrm{~s}$ vs. $300 \pm 20 \mathrm{~s}, p=0.26, \mathrm{HI} ; 295 \pm 22 \mathrm{~s}$ vs. $298 \pm 16 \mathrm{~s}, p=0.47)$, so the mean result from day 1 and day 9 were calculated to represent a control performance (no taper) for each participant. Performance measures data, dietary intake and physical activity data were analysed via a twoway repeated measures ANOVA, with Bonferroni post-hoc analysis. Accelerometer wear time was analysed for both conditions using a one-way repeated measures ANOVA. Magnitudebased inferences about the true (population) effect of the RP taper and HI taper on 1,500 m running performance were calculated. The uncertainty in the effect was expressed as $90 \%$ confidence limits and as the likelihood that the true value of the effect represents substantial change; harm or benefit (Batterham and Hopkins 2006). The smallest meaningful change (SMC) in $1,500 \mathrm{~m}$ performance was assumed to be a reduction or increase in running time of $1 \%$. Changes in performance time were expressed as multiples of the SMC and the magnitude was considered either small (1x), moderate (3x), large (6x) or very large (10x) (Buchheit 2016). For other variables, effect size (ES) was calculated and was considered either trivial $(0-0.19)$, small (0.20-0.49), medium (0.50-0.79) or large ( $\geq 0.80)$ (Cohen 1992). Mean daily physical activity and carbohydrate intake from the final three days of the taper in both conditions were 
compared using paired samples $t$-tests. Results are presented as mean \pm SD or $\pm 90 \%$ confidence interval (CI), unless stated otherwise. Statistical significance was accepted at $p \leq 0.05$.

\section{Results}

Training completed during control and taper periods in both conditions is presented in table 3 .

Outcome variables of the laboratory interval session are presented in table 4 .

Table 3. Training completed in the race-pace and high-intensity conditions and percentage change from control to taper periods

\begin{tabular}{|c|c|c|c|c|c|c|}
\hline \multirow[b]{2}{*}{ Training Variables } & \multicolumn{3}{|c|}{$\mathbf{R P}$} & \multicolumn{3}{|c|}{ HI } \\
\hline & Control & Taper & $\% \Delta$ & Control & Taper & $\% \Delta$ \\
\hline \multicolumn{7}{|l|}{ Training volume } \\
\hline Continuous running $(\mathrm{km})$ & $45 \pm 13$ & $33 \pm 9$ & $-27 \%$ & $41 \pm 15$ & $30 \pm 9$ & $-27 \%$ \\
\hline Interval running $(\mathrm{km})$ & $10 \pm 4$ & $6 \pm 2$ & $-45 \%$ & $9 \pm 4$ & $5 \pm 2$ & $-45 \%$ \\
\hline Total running (km) & $55 \pm 14$ & $38 \pm 9$ & $-30 \%$ & $50 \pm 15$ & $35 \pm 9$ & $-30 \%$ \\
\hline \multicolumn{7}{|l|}{ Training frequency } \\
\hline Continuous running (runs $\cdot$ week $^{-1}$ ) & $4 \pm 1$ & $4 \pm 0$ & $-15 \%$ & $4 \pm 1$ & $4 \pm 1$ & $-14 \%$ \\
\hline Interval running (runs·week ${ }^{-1}$ ) & $2 \pm 0$ & $2 \pm 0$ & $0 \%$ & $2 \pm 1$ & $2 \pm 1$ & $0 \%$ \\
\hline Total running (runs $\cdot$ week $^{-1}$ ) & $7 \pm 1$ & $6 \pm 0$ & $-10 \%$ & $7 \pm 2$ & $6 \pm 1$ & $-10 \%$ \\
\hline \multicolumn{7}{|l|}{ Training intensity } \\
\hline Continuous running ( $\%$ race speed) & $61 \pm 8$ & $57 \pm 9$ & $-7 \%$ & $62 \pm 8$ & $58 \pm 9$ & $-6 \%$ \\
\hline Interval running (\% race speed) & $89 \pm 8$ & $95 \pm 5$ & $+7 \%$ & $94 \pm 8$ & $99 \pm 6$ & $+5 \%$ \\
\hline Laboratory interval session (\% race speed) & - & $100 \pm 0$ & - & - & $115 \pm 0$ & - \\
\hline
\end{tabular}

Data are mean $\pm \mathrm{SD} ; n=10$; RP, race-pace condition; HI, high-intensity condition; $\% \Delta$, mean percentage change from control to taper periods. 
Table 4. Average speed, volume and psychophysiological responses during the controlled laboratory interval session in the race-pace and high intensity conditions

\begin{tabular}{lrrc}
\hline & \multicolumn{1}{c}{ RP } & \multicolumn{1}{c}{ HI } & $\boldsymbol{p}$ value \\
\hline Speed $\left(\mathrm{m} \cdot \mathrm{s}^{-1}\right)$ & $5.8 \pm 0.5$ & $6.6 \pm 0.6$ & 0.002 \\
Volume $(\mathrm{km})$ & $2.7 \pm 0.7$ & $2.7 \pm 0.7$ & N/A \\
Heart rate $\left(\mathrm{b} \cdot \mathrm{min}^{-1}\right)$ & $169.0 \pm 9.0$ & $178.0 \pm 7.0$ & 0.001 \\
RPE & $14.0 \pm 1.0$ & $17.0 \pm 1.0$ & 0.001 \\
Peak lactate $\left(\mathrm{mmol} \cdot \mathrm{L}^{-1}\right)$ & $3.8 \pm 1.6$ & $9.9 \pm 3.4$ & 0.001 \\
\hline
\end{tabular}

Data are mean \pm SD; significance determined by paired samples $t$-test $(n=10)$; RP, race-pace condition; HI, highintensity condition.

\section{1,500 m performance assessment}

The change in performance times after tapering for the RP and HI conditions are shown in figure 2A. When considered relative to the SMC in performance, qualitative inference suggests that the RP tapering strategy was most likely to be beneficial to $1,500 \mathrm{~m}$ time $(\mathrm{SMC}=3.0 \mathrm{~s}, \mathrm{CI}$ $=8.5$ to $11.7 \mathrm{~s}$, with chances of a beneficial/trivial/harmful effect being $100 \%, 0 \%$ and $0 \%$ ), whereas the HI taper was unclear $(\mathrm{SMC}=3.0 \mathrm{~s}, \mathrm{CI}=-7.8$ to $16.2 \mathrm{~s}$, with chances of a beneficial/trivial/harmful effect being 57\%, 29\% and 14\%; table 5). Individual responses to RP and $\mathrm{HI}$ conditions are shown in figures $2 \mathrm{~B}$ and $2 \mathrm{C}$, respectively. There was a main effect of taper (control training vs. taper training, $p=0.001)$ on peak blood lactate $(\mathrm{RP} ; 7.1 \pm 3.1$ $\mathrm{mmol} \cdot \mathrm{L}^{-1}$ vs. $10.1 \pm 2.6 \mathrm{mmol} \cdot \mathrm{L}^{-1}, \mathrm{ES}=1.08, \mathrm{HI} ; 7.7 \pm 2.4 \mathrm{mmol} \cdot \mathrm{L}^{-1}$ vs. $9.3 \pm 2.5 \mathrm{mmol} \cdot \mathrm{L}^{-1}$, $\mathrm{ES}=0.63$ ), with no difference in peak heart rate between strategies (control vs. taper; $p=0.79$, $\mathrm{RP} ; 182 \pm 7$ b. $\min ^{-1}$ vs. $184 \pm 9$ b. $\min ^{-1}, \mathrm{ES}=0.20, \mathrm{HI} ; 184 \pm 8$ b. $\mathrm{min}^{-1}$ vs. $183 \pm 7$ b. $\mathrm{min}^{-1}$, ES $=0.13)$ or conditions (RP vs. HI; $p=0.43)$. 

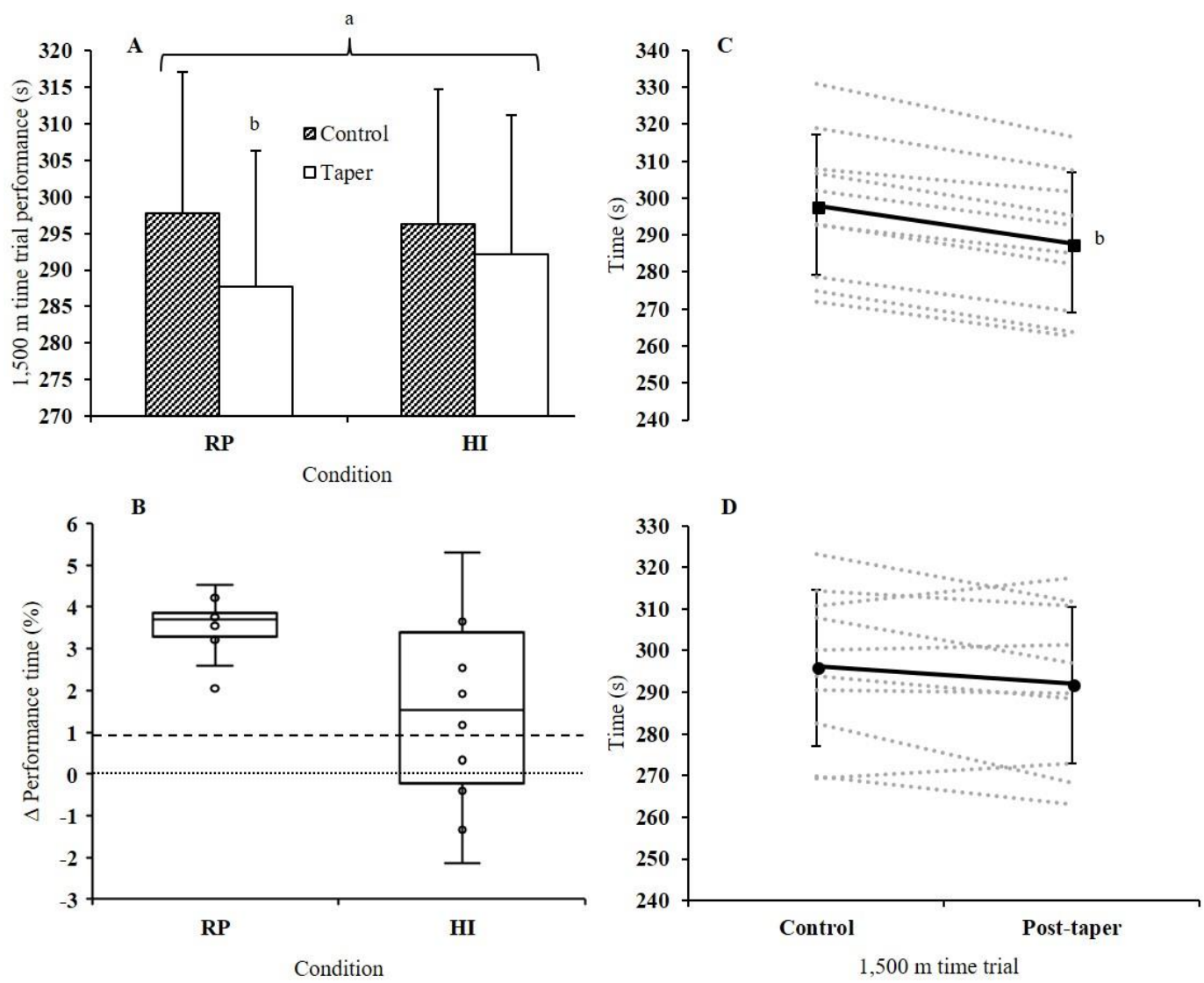

Figure 2. Change in performance time (\%) after tapering compared to control in RP and HI (A). Circles represent individual responses, median response shown as horizontal line within the box. Positive values represent an improvement in performance, negative values represent a worsening in performance. No change in performance (dotted line), smallest meaningful change in 1,500 m treadmill time trial performance measure (1\%, dashed line). Individual 1,500 $m$ time trial performance responses (dashed lines) and group mean $\pm S D$ (solid line) in $R P(\boldsymbol{B})$ and $H I(\boldsymbol{C})$. 
Table 5. Differences in pre- and post-taper 1,500 $\mathrm{m}$ time trial performance

\begin{tabular}{lcccc}
\hline & $\begin{array}{c}\text { Pre-taper Time } \\
(\mathbf{s})\end{array}$ & $\begin{array}{c}\text { Post-taper } \\
\text { Time (s) }\end{array}$ & $\begin{array}{c}\text { Mean Improvement (s) and } \\
\mathbf{9 0 \%} \mathbf{C L}\end{array}$ & $\begin{array}{c}\text { Factor of the Smallest } \\
\text { Important Effect }^{a}\end{array}$ \\
\hline $\mathrm{RP}$ & $298.8 \pm 19.3$ & $288.7 \pm 18.5$ & $10.1 ; \pm 1.6$ & $3.4^{* * * * *}$ \\
$\mathrm{HI}$ & $296.3 \pm 18.4$ & $292.1 \pm 19.1$ & $4.2 ; \pm 12.0$ & $1.4^{*}$ \\
\hline
\end{tabular}

Data are mean $\pm S D$ unless stated otherwise; $n=10 ;{ }^{a}$, with reference to a smallest worthwhile change of $1 \%$;

The numbers of asterisks (*) indicate the likelihood for differences to be substantial, with 1 symbol referring to possible difference, 2 to likely, 3 to very likely, and 4 to most likely; RP, race-pace condition; HI, high-intensity condition; $C L$, confidence limits.

\section{Force measurement}

A main effect of tapering was evident for MVC force $(p=0.002)$ and for RFD $(p=0.02)$. There was a main effect of time on RFD ( $\left.\mathrm{F}_{50} v s . \mathrm{F}_{100} v s . \mathrm{F}_{150}, p=0.001\right)$. No interaction effect on MVC force was observed (RP; $722.3 \pm 149.9$ vs. $663.4 \pm 153.1 \mathrm{~N},+9 \%, \mathrm{ES}=0.39, \mathrm{HI} ; 721.7$ $\pm 143.3 v s .682 .3 \pm 130.2 \mathrm{~N},+6 \%, \mathrm{ES}=0.29, p=0.40$ ), or a condition $x$ time interaction effect for RFD (RP; $\mathrm{ES}=0.54,0.50,0.56, \mathrm{HI}$; $\mathrm{ES}=0.22,0.34,0.22$ for $\mathrm{F}_{50}, \mathrm{~F}_{100}, \mathrm{~F}_{150}$, respectively, $p=0.06)$.

\section{Dietary intake and physical activity}

Mean daily energy intake remained consistent throughout both conditions (RP; $2907 \pm 419$ vs. $2812 \pm 506 \mathrm{kcal}, \mathrm{ES}=0.21, \mathrm{HI} ; 2815 \pm 366$ vs. $2728 \pm 456 \mathrm{kcal}, \mathrm{ES}=0.21, p=0.16$ ). A main effect of tapering was evident $(p=0.001)$ when mean daily carbohydrate consumption was expressed relative to mean daily running volume $(\mathrm{km})$, suggesting a daily carbohydrate excess during tapering compared to control, but without differences between conditions ( $p=0.94)$. There was no change in body mass throughout both conditions (RP; $71.9 \pm 7.0 \mathrm{~kg} v s .72 .0 \pm$ $6.9 \mathrm{~kg}, \mathrm{ES}=0.01, p=0.70, \mathrm{HI} ; 73.1 \pm 6.5 \mathrm{~kg} v s .72 .9 \pm 6.8 \mathrm{~kg}, \mathrm{ES}=0.04, p=0.25)$. 
Daily physical activity (counts. $\min ^{-1}$ ) was lower during tapering compared to the control period (main effect of tapering; $p=0.04$ ). Time spent in MVPA was lower during tapering compared to control (main effect of tapering; $-1.6 \%, p=0.03$ ). There was no difference in time spent sedentary or in light physical activity between strategies (control vs. taper; $p=0.71$ and $p=0.66$, respectively) or conditions (RP $v s$. HI; $p=0.55$ and $p=0.86$ ).

In the last three days of the taper after the laboratory interval session, there were no differences in physical activity (counts $\cdot \min ^{-1}, \mathrm{ES}=0.48, p=0.57$ ) or sedentary time $(\mathrm{ES}=$ $0.10, p=0.25)$, in light $(\mathrm{ES}=0.18, p=0.09)$, or in MVPA $(\mathrm{ES}=0.25, p=0.79)$ between $\mathrm{RP}$ and HI. Mean daily carbohydrate intake was consistent in both conditions ( $\mathrm{ES}=0.18, p=0.34)$.

\section{Discussion}

In sub-elite runners, $1,500 \mathrm{~m}$ performance was improved by $3.4 \%$ after a tapering protocol where the final interval session was completed at race pace. The small $90 \% \mathrm{CI}$ indicated that participants responded similarly to this strategy. The effect of completing the final interval session at $115 \%$ of race pace is possibly beneficial (1.4\%), although there was large variation in individual responses, with some runners improving performance and others experiencing a worsening in performance.

Improvements of between 0.5-6.0\% are expected following a successful taper (Mujika and Padilla 2003). In a meta-analysis of the available literature, Bosquet et al. (2007) reported a mean performance improvement of $1.96 \%$ in competitive runners, swimmers and cyclists. In runners specifically, improvements in time trial or actual race performance have been reported in the range of 1.6-3.0\% (Houmard et al. 1994, Munoz et al. 2015). The observed improvement in performance from the RP taper (3.4\%) falls within the expected range and was most likely to have a positive effect on performance. Whilst the effect of the HI taper on performance at the group level was unclear, performance time after HI was improved in six out of ten 
individuals in excess of the SMC in performance (1\%). In one individual, there was a greater in improvement in performance after $\mathrm{HI}$ compared to RP and this was also the largest improvement from baseline (5\%) across both conditions.

This was not the case in all individuals and the $90 \%$ confidence limits indicate that negative results were experienced by some athletes implementing the HI taper. The $1^{\text {st }}, 6^{\text {th }}$ and $8^{\text {th }}$ fastest participants experienced a worsening in performance time after this condition, which also demonstrates that individual responses did not appear to be related to performance standard (i.e., performance times in the control time trial). Although a greater capacity to respond effectively to high intensity training during the taper has been suggested (Shepley et al. 1992, Houmard et al. 1994, Mujika et al. 2004), the conservative reduction in training volume resulting from the prediction models may not have allowed sufficient recovery for most individuals to respond positively to the increased intensity of this session. The $\sim 30 \%$ reduction in overall volume represents a comparatively small adjustment compared to other studies where volume is reduced by up to $\sim 90 \%$ and wholly dedicated to high intensity training (Shepley et al. 1992, Houmard et al. 1994). The volume reduction and duration of the taper (7 d) was also less than the $41-60 \%$ reduction over two weeks recommended from a meta-analysis on the effects of tapering on performance (Bosquet et al. 2007). However, existing experimental research on tapering typically focuses on improving a single performance and does not consider that elite endurance athletes often need to perform in multiple competitions in the build up to their major championship. This may explain the reason for not reducing volume substantially immediately prior to competition, due to a lengthy peaking period and the need to maintain fitness (Solli et al, 2017, Tønnessen et al. 2014). Nevertheless, the algorithm-derived taper was not designed for manipulation of training intensity above race speed, therefore a concomitant decrease in volume over a longer taper duration might be necessary to optimise performance using this strategy. 
The volume of the final interval session itself was greater than $1,500 \mathrm{~m}$ race distance $(2.7 \pm 0.7 \mathrm{~km})$, due to tapered training being prescribed relative to regular training, which was uncontrolled prior to the study. Whilst this was the case for both conditions, a session of this volume completed faster than race pace may have exacerbated fatigue in some individuals, with insufficient recovery time before the performance assessment. This may have been attenuated by increasing the recovery time between $300 \mathrm{~m}$ repetitions in the final interval session in HI, to compensate for the increase in intensity. Alternatively, the increase in intensity of $15 \%$ above race pace may have been too aggressive for some individuals to respond positively to, given the close proximity of performance assessment.

In the days after the final interval session, participants did not modify their overall physical activity or training in HI compared to RP. This suggests they did not spend more time resting to compensate for the increased training stress. It may be that some athletes require longer to reach peak performance after the final interval session in HI, perhaps due to the additional training stress and in light of insufficient recovery. It was not possible however, to explore the amplitude of the performance rebound between the RP and HI strategies, since participants completed one performance assessment after each taper condition.

The uncontrolled prior training load may have contributed to the worsening in performance after $\mathrm{HI}$ in some individuals. It has been observed previously that deliberate overload/overreaching can result in greater performance super-compensation compared to habitual training, providing that the training stress from overload does not exceed capacity to recover during the taper (Le Meur et al. 2013, Aubry et al. 2014). Only one week of control training data was collected to inform the taper prescription and this may not have been representative of the extent to which athletes were undergoing sub-optimal, habitual or overloaded training prior to the study. The addition of a higher intensity interval session may have influenced capacity to recover during the taper in some individuals in HI if they were 
overreaching beforehand, particularly given the volume and intensity of this session. A more thorough method to monitor training load that incorporates internal load prior to, and during the taper, might have added to understanding of individual responses.

The timing of the non-laboratory interval sessions may also have influenced the ability to recover in some individuals. For example, participants who were prescribed a frequency of three interval sessions during the taper may have been programmed to complete non-laboratory interval sessions on consecutive days, in order to protect the controlled interval session and allow a rest day before the final time trial.

Differences in the performance changes from each condition (RP vs. HI) cannot be explained by the measured physiological indices. However, there were several main effects of tapering (control vs. taper). Peak blood lactate after the performance trial increased in both conditions after tapering compared to control. This suggests that a greater contribution to energy production from glycolysis occurred after tapering and supports the important role of glycolytic metabolism in middle-distance running performance (Stellingwerff et al. 2011). A consistently reported physiological response to tapering is an increase in muscle glycogen concentration (Shepley et al. 1992, Neary et al. 1992), which may facilitate increased glycolytic energy contribution and maximal performance capability (Houmard et al. 1994, Mujika et al. 2004). Although muscle glycogen was not measured in the current study, carbohydrate consumption remained consistent despite a reduction in overall physical activity and a lower proportion of time in MVPA during the taper. This may reflect an increase in muscle glycogen, as shown previously following a 7-d taper (Shepley et al. 1992). In the present study, there was no change in carbohydrate consumption in the final three days of HI compared to RP, despite an increase in intensity of the final interval session. Since glycogen is the main energy source for high intensity exercise (Hermansen et al. 1967, Romijn et al. 1993, Hargreaves 1997, Stellingwerff et al. 2011) and there is evidence of muscle glycogen depletion in type II fibres 
after high intensity intermittent exercise (Gollnick et al. 1973), a more direct intervention to optimise carbohydrate consumption after the intensified interval session in HI might have influenced the performance outcome.

Both MVC force and RFD were improved after tapering, supporting previous research in swimmers (Cavanaugh and Musch 1989, Costill et al. 1985, Johns et al. 1992, Raglin et al. 1996). Improvements in peak force and absolute power of the single muscle fibre have also been observed alongside improvements in performance after tapering in endurance runners (Luden et al. 2010). These parameters have been shown to respond to the reduced training load during the taper, potentially due to attenuation in function during periods of intensive training

rather than an improvement per se. Since there were no differences in the improvement of MVC force and RFD between conditions, this further supports the notion that improvements in running performance after tapering are influenced by a multitude physiological factors. Although not measured in the present study, other physiological mechanisms associated with tapering may include; improvements in maximal oxygen uptake and running economy, positive changes in haematology, and a hormonal milieu favourable to anabolic processes (Mujika et al. 2004).

\section{Limitations}

The participants in the present study were sub-elite and heterogeneous for performance level, which may limit the application of findings to elite athletes. However, performance level did not appear to explain the individual differences in response to the HI taper. Assessment of the physiological characteristics of the participants may have facilitated our understanding and interpretation of the individual responses, although this was not feasible in the present study. It is likely that between-athlete variability exists in how performance is generated, particularly in middle-distance running (Sandford et al. 2018). Two athletes with similar $1,500 \mathrm{~m}$ 
performance times for example, might therefore elicit different responses to a fixed intensity session at $115 \%$ of race pace, owing to potential differences in maximal speed, aerobic and anaerobic capacities.

Whilst participants were instructed to repeat control training from the first condition in the control period of the second condition, GPS data revealed slight discrepancies between control training loads in RP versus HI. However, the taper in both conditions was prescribed relative to the training completed in the corresponding control periods and therefore slight differences were accounted for by the prediction models.

The 1,500 m performance assessments were completed on an 'on response' treadmill to eliminate environmental influences and for the feasibility of carrying out additional measurements. However, the performance times of all participants in the current study were considerably slower than their personal bests, likely owing to numerous factors related to the controlled nature of the performance measure, including; lack of competition/opponent, time feedback and verbal encouragement, and the responsiveness of the treadmill to autonomous speed adjustment. The study also took place earlier than the athletes' typical peaking phase for the summer track season. Participants were not informed about the precise differences between the two tapering strategies, but they could not be blinded to manipulation of the training load. It is therefore unknown whether their preconceptions about the training they completed prior to the post-taper performance assessments may have influenced the outcome.

\section{Conclusion}

A 7-d taper prescribed using prediction models based on the current practices of elite British middle-distance runners is most likely to improve $1,500 \mathrm{~m}$ treadmill time trial performance (3.4\%) in young, sub-elite runners. Performance may possibly be improved (1.4\%) by running $15 \%$ faster than race pace in the final interval session of this taper, but this strategy should be 
attempted with caution, due to highly variable effects on performance. To increase the likelihood of improving performance after this strategy, a greater reduction in overall training volume may be required, or adjustments to the prescription of the final interval session in terms of volume, intensity or duration of recovery intervals. Further research is required to investigate this, and in relation to track running performance.

\section{Acknowledgements}

The authors would like to thank the English Institute of Sport, the Loughborough University Sports Technology Institute, Dr Adam Fry and Dr Andy Jackson for their support. The authors would also like to acknowledge Kirsty Addy, Shauna Chambers and Nicola Rawlinson for their assistance with data collection.

\section{Conflicts of interest \& sources of funding}

The authors declare that they have no conflicts of interest and the study did not receive funding from sources external to Loughborough University. 


\section{References}

Allison, G.T. 2003. Trunk muscle onset detection technique for EMG signals with ECG artefact. J. Electromyogr. Kinesiol. 13(3): 209-216. doi: 10.1016/S1050-6411(03)00019-1.

Aubry, A., Hausswirth, C., Louis, J., Coutts, A.J., and Le Meur, Y. 2014. Functional overreaching: the key to peak performance during the taper? Med. Sci. Sports Exerc. 46(9): 1769-1777. doi: 10.1249/MSS.0000000000000301.

Batterham, A.M., and Hopkins, W.G. 2006. Making meaningful inferences about magnitudes. Int. J. Sports Physiol. Perform. 1(1): 50-57. doi: 10.1123/ijspp.1.1.50.

Berryman, N., Mujika, I., Arvisais, D., Roubeix, M., Binet, C., and Bosquet, L. 2018. Strength training for middle-and long-distance performance: a meta-analysis. Int. J. Sport. Physiol. 13(1): 57-64. doi: 10.1123/ijspp.2017-0032.

Blagrove, R.C., Howatson, G., and Hayes, P.R. 2017. Effects of strength training on the physiological determinants of middle-and long-distance running performance: a systematic review. Sports Med. 48(5): 1117-1149. doi: 10.1007/s40279-017-0835-7.

Bosquet, L., Montpetit, J., Arvisais, D., and Mujika, I. 2007. Effects of tapering on performance: A meta-analysis. Med. Sci. Sports Exerc. 39(8): 1358-1365. doi: 10.1249/mss.0b013e31806010e0.

Buchheit, M. 2016. The numbers will love you back in return-I promise. Int. J. Sports Physiol. Perform. 11(4): 551-554. doi: 10.1123/ijspp.2016-0214.

Buckthorpe, M.W., Hannah, R., Pain, T.G., and Folland, J.P. 2012. Reliability of neuromuscular measurements during explosive isometric contractions, with special reference to electromyography normalization techniques. Muscle Nerve. 46(4): 566-576. doi: 10.1002/mus.23322.

Cavanaugh, D. and Musch, K. 1989. Arm and leg power of elite swimmers increase after taper as measured by biokinetic variable resistance machines. J. Swim. Res. 5(3): 7-10. 
Cohen, J. 1992. A power primer. Psychological Bulletin. 112(1): 155-159. doi: 10.1037/00332909.112.1.155.

Costill, D.L., King, D., Thomas, R., and Hargreaves, M. 1985. Effects of reduced training on muscular power in swimmers. Phys. Sports Med. 13(2): 94-101. doi: 10.1080/00913847.1985.11708748.

Freedson, P.S., Melanson, E., and Sirard, J. 1998. Calibration of the computer science and applications, inc. accelerometer. Med. Sci. Sports Exerc. 30(5): 777-781. doi: 10.1097/00005768-199805000-00021.

Gollnick, P.D., Armstrong, R.B., Sembrowich, W.L., Shepherd, R.E., and Saltin, B. 1973. Glycogen depletion pattern in human skeletal muscle fibres after heavy exercise. J. Appl. Physiol. 34(5): 615-618.

Halson, S.L., and Jeukendrup, A.E. 2004. Does overtraining exist? Sports Med. 34(14): 967981. doi: 10.2165/00007256-200434140-00003.

Hargreaves, M. 1997. Interactions between muscle glycogen and blood glucose during exercise. Exerc. Sport Sci. Rev. 25 (1): 21-39.

Hermansen, L., Hultman, E., and Saltin, B. 1967. Muscle glycogen during prolonged severe exercise. Acta Physiol. Scand. 71(2-3): 129-139. doi: 10.1111/j.1748-1716.1967.tb03719.x.

Hodges, K., Hancock, S., Currell, K., Hamilton, B., and Jeukendrup, A.E. 2006. Pseudoephedrine enhances performance in 1500-m runners. Med. Sci. Sports Exerc. 38(2): 329-333. doi: 10.1249/01.mss.0000183201.79330.9c.

Hopkins, W.G. 2005. Competitive performance of elite track and-field athletes: variability and smallest worthwhile enhancements. Sportscience. 9: 17-20.

Houmard, J.A. 1991. Impact of reduced training on performance in endurance athletes. Sports Med. 12(6): 380-393. doi: 10.2165/00007256-199112060-00004. 
Houmard, J.A., Scott, B.K., Justice, C.L., and Chenier, T.C. 1994. The effects of taper on performance in distance runners. Med. Sci. Sports Exerc. 26(5): 624-631. doi: 07959131/94/2605-0624.

Johns, R.A., Houmard, J.A., Kobe, R.W., et al. 1992. Effects of taper on swim power, stroke distance, and performance. Med. Sci. Sports Exerc. 24(10): 1141-1146.

Jones, A.M., and Doust, J.H. 1996. A $1 \%$ treadmill grade most accurately reflects the energetic cost of outdoor running. J. Sports Sci. 14(4): 321-327. doi: 10.1080/02640419608727717.

Le Meur, Y., Pichon, A., Schaal, K., et al. 2013. Evidence of parasympathetic hyperactivity in functionally overreached athletes. Med. Sci. Sports Exerc. 45(11): 2061-2071. doi: 10.1249/MSS.0b013e3182980125.

Luden, N., Hayes, E., Galpin, A., et al. 2010. Myocellular basis for tapering in competitive distance runners. J. Appl. Physiol. 108(6): 1501-1509. doi: 10.1152/japplphysiol.00045.2010. Mujika, I. 1998. The influence of training characteristics and tapering on the adaptation in highly trained individuals: A review. Int. J. Sports Med. 19(7): 439-446. doi: 10.1055/s-2007971942.

Mujika, I. 2010. Intense training: the key to optimal performance before and during the taper. Scand. J. Med. Sci. Sports. 20(s2): 24-31. doi: 10.1111/j.1600-0838.2010.01189.x Mujika, I., and Padilla, S. 2000. Detraining: loss of training-induced physiological and performance adaptations. Part I. Sports Med. 30(2): 79-87. doi: 10.2165/00007256200030020-00002.

Mujika, I., and Padilla, S. 2003. Scientific bases for precompetition tapering strategies. Med. Sci. Sports Exerc. 35(7): 1182-1187. doi: 10.1249/01.MSS.0000074448.73931.11.

Mujika, I., Padilla, S., Pyne, D., and Busso, T. 2004. Physiological changes associated with the pre-event taper in athletes. Sports Med. 34(13): 891-927. doi: 10.2165/00007256-20043413000003. 
Munoz, I., Seiler, S., Alcocer, A., Carr, N., \& Esteve-Lanao, J. 2015. Specific intensity for peaking: is race pace the best option? Asian J. Sports Med. 6(3): e24900. doi: 10.5812/asjsm.24900.

Neary, J.P., Martin, T.P., Reid, D.C., Burnham, R., and Quinney, H.A. 1992. The effects of a reduced exercise duration taper programme on performance and muscle enzymes of endurance cyclists. Eur. J. Appl. Physiol. 65(1): 30-36. doi: 10.1007/BF01466271.

Raglin, J.S., Koceja, D.M., Stager, J.M., and Harms, C.A. 1996. Mood, neuromuscular function, and performance during training in female swimmers. Med. Sci. Sports Exerc. 28(3): 372-377. doi: 10.1097/00005768-199603000-00013.

Romijn, J.A., Coyle, E.F., Sidossism L.S., et al. 1993. Regulation of endogenous fat and carbohydrate metabolism in relation to exercise intensity and duration. Am. J. Physiol. Endocrinol. Metab. 265(3): E380-E391. doi: 10.1152/ajpendo.1993.265.3.E380.

Sandford, G.N., Allen, S.V., Kilding, A.E., Ross, A., and Laursen, P.B. 2018. Anaerobic speed reserve: a key component of elite male 800m running. Int. J. Sports Physiol. Perform. In press. doi: 10.1123/ijspp.2018-0163.

Shepley, B., MacDougall, J.D., Cipriano, N., Sutton, J.R., Tarnopolsky, M.A., and Coates, G. 1992. Physiological effects of tapering in highly trained athletes. J. Appl. Physiol. 72(2): 706711. doi: 10.1152/jappl.1992.72.2.706.

Solli, G.S., Tønnessen, E., and Sandbakk, Ø. 2017. The training characteristics of the world's most successful female cross-country skier. Front. Physiol. 8: 1069. doi: 10.3389/fphys.2017.01069.

Spilsbury, K.L., Fudge, B.W., Ingham, S.A., Faulkner, S.H., and Nimmo, M.A. 2015. Tapering strategies in elite British endurance runners. Eur. J. Sport Sci. 15(5): 367-373. doi: 10.1080/17461391.2014.955128. 
Stellingwerff, T. 2012. Case study: nutrition and training periodisation in three elite marathon runners. Int. J. Sports. Nutr. Exerc. Metab. 22(5): 392-400. doi: 10.1123/ijsnem.22.5.392.

Stellingwerff, T., Maughan, R.J., and Burke, L.M. 2011. Nutrition for power sports: middledistance running, track cycling, rowing, canoeing/kayaking, and swimming. J. Sports Sci. 29(sup1): S79-89. doi: 10.1080/02640414.2011.589469.

Thomas, L., Mujika, I., and Busso, T. Computer simulations assessing the potential performance benefit of a final increase in training during pre-event taper. 2009. J. Strength Cond. Res. 23(6): 1729-1736. doi: 10.1519/JSC.0b013e3181b3dfa1.

Tillin, N.A., Jimenez-Reyes, P., Pain, M.T., and Folland, J.P. 2010. Neuromuscular performance of explosive power athletes versus untrained individuals. Med. Sci. Sports Exerc. 42(4): 781-790. doi: 10.1249/MSS.0b013e3181be9c7e.

Tillin, N.A., Pain, M.T., and Folland, J.P. 2013. Identification of contraction onset during explosive contractions. Response to Thompson et al. "Consistency of rapid muscle force characteristics: influence of muscle contraction onset detection methodology". J. Electromyogr. Kinesiol. 23(4): 991-994. doi: 10.1016/j.jelekin.2013.04.015.

Tønnessen, E., Sylta, Ø., Haugen, T.A., Hem, E., Svendsen, I.S., and Seiler, S. 2014. The road to gold: training and peaking characteristics in the year prior to a gold medal endurance performance. PloS One. 9(7): e101796.

Wiles, J.D., Bird, S.R., Hopkins, J., and Riley, M. 1992. Effect of caffeinated coffee on running speed, respiratory factors, blood lactate and perceived exertion during 1500-m treadmill running. Br. J. Sports Med. 26(2): 116-20. doi: 10.1136/bjsm.26.2.116. 